\title{
Kernos
}

Revue internationale et pluridisciplinaire de religion grecque antique

$23 \mid 2010$

Varia

\section{Richard BUXTON, Forms of Astonishment. Greek Myths of Metamorphosis}

\section{Hélène Collard}

\section{OpenEdition \\ Journals}

Édition électronique

URL : http://journals.openedition.org/kernos/1646

DOI : 10.4000/kernos. 1646

ISSN : 2034-7871

\section{Éditeur}

Centre international d'étude de la religion grecque antique

Édition imprimée

Date de publication : 1 janvier 2010

Pagination : 395-397

ISSN : 0776-3824

Référence électronique

Hélène Collard, «Richard вuxton, Forms of Astonishment. Greek Myths of Metamorphosis », Kernos [En ligne], 23 | 2010, mis en ligne le 15 septembre 2011, consulté le 21 septembre 2020. URL : http:// journals.openedition.org/kernos/1646; DOI : https://doi.org/10.4000/kernos.1646

Ce document a été généré automatiquement le 21 septembre 2020.

Kernos 


\title{
Richard BUXTON, Forms of Astonishment. Greek Myths of Metamorphosis
}

\author{
Hélène Collard
}

\section{RÉFÉRENCE}

Richard BuxToN, Forms of Astonishment. Greek Myths of Metamorphosis, Oxford, Oxford University Press, 2009. 1 vol. 14,5 × 22,5 cm, xvi + 281 p. ISBN : 978-0-19-924549-9.

1 Comme le laisse entendre le sous-titre, ce travail est une réflexion sur les mythes grecs de métamorphose. Dans les récits, il n'est pas rare en effet qu'un être, humain ou divin, subisse une transformation, quelle qu'elle soit. R. Buxton propose ici de se pencher sur ce phénomène. Dès l'introduction, après un rapide état de la question, l'A. précise la portée de son étude : il entend partir des travaux antérieurs consacrés au même sujet ${ }^{1}$, mais en essayant d'aller plus loin que ses prédécesseurs dans l'analyse. Il emmène ainsi le lecteur dans un formidable voyage, explorant de nombreux récits grecs dans lesquels la métamorphose intervient. Toutefois, il ne se limite pas là, son but étant de faire avancer notre compréhension de ce phénomène particulier et de la signification qu'il a pu avoir pour les Grecs. Il nous invite donc, au fil de l'analyse, à nous interroger sur certains aspects de la culture et de la religion grecques, notamment sur la catégorisation des êtres vivants - dieux, humains, animaux, plantes - et sur les chevauchements possibles entre ces différentes classes, ou encore sur la notion d'anthropomorphisme divin.

$2 \mathrm{Si}$, en plusieurs occasions, l'A. fait référence à des images, son travail se fonde principalement sur les textes, comme il l'annonce d'emblée (p. 22). Il commence d'ailleurs son étude par l'inventaire des différents mots utilisés pour évoquer la métamorphose. De fait, on constate que le terme metamorphôsis n'apparaît que rarement, et ce dans des textes tardifs. Les auteurs ont donc eu recours à d'autres 
groupes de mots pour exprimer l'idée de changement ou de transformation, pour parler de l'apparence physique, mais aussi pour décrire les effets de la métamorphose. En effet, ils se sont souvent attachés à relater la réaction des témoins de ces transformations, qu'ils en soient les victimes ou de simples spectateurs. Le terme qu'on retrouve alors le plus souvent est thambos, qui reflète un sentiment d'étonnement, de stupéfaction et d'admiration dû à l'irruption soudaine du sacré dans le quotidien des humains, et qui peut être traduit en anglais par astonishment, mot qu'on retrouve dans le titre de l'ouvrage. Cette notion tiendra donc une place importante tout au long de l'analyse, et l'un des objectifs de l'A. sera de répertorier et de discuter la présence du thambos, ou son absence. Car si la métamorphose provoque généralement la surprise, ce n'est pas toujours le cas.

L'ouvrage se divise ensuite en deux parties, composées chacune de cinq chapitres. La première partie se charge d'étudier les mythes de métamorphose considérés dans leur contexte narratif, et ce dans un ordre chronologique. Le chapitre 1 est ainsi consacré à l'épopée homérique, et surtout à l'odyssée, où les passages relatant une transformation sont nombreux. Dans le chapitre 2, c'est la tragédie et la comédie de l'Athènes classique qui sont au centre des préoccupations. Les chapitres 4 et 5 se penchent sur les textes hellénistiques pour l'un, notamment les Argonautiques d'Apollonios de Rhodes et l' Europe de Moschos, et post-hellénistiques pour l'autre, principalement les Dionysiaques de Nonnos. Chaque passage évoqué est illustré par un ou plusieurs extraits, et les termes disant la métamorphose sont toujours retranscrits en grec. L'étude des mots se poursuit donc tout au long de l'ouvrage. L'A. se penche ensuite sur les caractéristiques propres à chaque auteur, à chaque période et à chaque genre, pour comprendre comment le contexte a pu façonner la manière de raconter ces histoires. Il tente également de répondre à une question qui a continuellement préoccupé les modernes : dans quelle mesure les récits de transformation étaient-ils pris au sérieux? Ce qui l'amène à s'interroger sur les traductions qui ont été faites de ces passages, et qui trahissent souvent le souci des exégètes de rendre la croyance en la métamorphose plus acceptable, en détournant le sens de certains mots, par exemple. Il est donc indispensable de retourner aux textes grecs et de se débarrasser de tout cet arrièreplan interprétatif pour rester objectif.

Le chapitre 3 diffère légèrement des quatre autres puisqu'il est consacré aux arts visuels. Que l'on me permette de m'y arrêter un peu. L'A. tente ici de dégager les différentes stratégies mises en œuvre par les artistes qui ont tenté de représenter ou de faire allusion à la métamorphose. En effet, ceux-ci se sont retrouvés face à des défis particuliers, différents de ceux des poètes ou des narrateurs. Il est facile de décrire avec des mots le fait que Zeus se soit transformé en taureau pour enlever Europe, mais comment rendre ce phénomène iconographiquement? Comment représenter en une seule image une divinité qui change de forme successivement, comme le dieu-fleuve Achéloos par exemple? Comment illustrer la métamorphose d'Actéon? Les images ne sont pas très nombreuses, peut-être en raison de ces difficultés justement, mais elles témoignent d'une grande richesse dans la diversité des solutions adoptées par les peintres. Dans certains cas, la métamorphose est déjà complète, dans d'autres, elle est en cours, ce qui est évoqué visuellement par un être « hybride ». C'est alors souvent le contexte général de l'image et la référence à l'arrière-plan mythique qui permettent au spectateur d'identifier le processus de transformation. Toutefois, ce serait une grosse erreur de considérer les images comme de simples dérivés des récits mythologiques. Au contraire, on peut dégager une tradition iconographique indépendante des textes, 
comme le montrent les représentations de l'épisode de Circé par exemple, qui vont bien au-delà de leur référent homérique. On comprend alors toute l'importance d'intégrer la production visuelle dans ce type d'étude.

5 Après cette première étape de mise en contexte, l'A. propose d'examiner un certain nombre d'éléments constitutifs de la tradition métamorphique dans le but de dégager, peut-être, des modèles récurrents. Le chapitre 6, intitulé " Shapes of the Gods », étudie la relation entre métamorphose et notion grecque de divinité, au moyen de divers questionnements sur les raisons qui pouvaient amener les dieux à changer de forme, sur l'étonnement que suscitait leur auto-transformation et sur la problématique de l'anthropomorphisme. Les deux chapitres suivants sont consacrés aux transformations de mortels, soit en éléments du paysage (chapitre 7), soit en éléments végétaux (chapitre 8). Ces histoires posent la question du statut de l'être humain par rapport au monde qui l'entoure. Le chapitre 9 tente d'éclaircir la manière dont les mythes de métamorphose étaient vus par leurs contemporains, au travers de la vision des philosophes surtout. On remarque ainsi que ces récits ont suscité des réactions variées de la part des penseurs, allant de la crédulité au scepticisme et même au rejet total. Le chapitre 10, qui fait office de conclusion, revient sur l'importance d'une approche contextuelle, tout en invitant à la prudence. S'il faut sans aucun doute tenir compte du contexte dans la démarche interprétative, il faut le faire de façon appropriée.

Les récits de métamorphose, qui peuvent paraître étranges et incroyables aux yeux du lecteur moderne, explorent les frontières entre le quotidien et le sacré, l'humain et le divin, l'animé et l'inanimé, mais surtout les points de contact entre ces catégories généralement considérées comme distinctes. Forms of Astonishment, à travers un sujet d'étude précis, soulève donc une série de questions pertinentes pour la compréhension des aspects généraux de la culture grecque, et c'est ce qui en fait son grand intérêt. Mentionnons encore la présence d'un index à la fin du livre et la fréquence des comparaisons avec d'autres traditions religieuses, anciennes et modernes, mais aussi avec la littérature, la science et même le cinéma. Je recommande donc vivement cet ouvrage passionnant à tous ceux qui s'intéressent à la religion grecque, et plus particulièrement à la représentation que les Grecs se faisaient du divin, du monde en général et de leur place au sein de celui-ci.

\section{NOTES}

1. Il s'agit principalement des deux ouvrages suivants : P.M.C. FORBES IRVING,Metamorphosis in Greek Myths, Oxford, 1990 et Fr. FRONTISI-DUCROUX, L'homme-cerf et la femme-araignée. Figures grecques de la métamorphose, Paris, 2003. 


\section{AUTEURS}

HÉLÈNE COLLARD

Université de Liège 